\title{
Time to culture conversion in smokers with pulmonary tuberculosis
}

\author{
J.A. Gullón1, I. Suárez11, M. Lecuona², R. Fernández1, G. Rubinos¹, \\ A. Medina1, C. Cabrera1', I. González1
}

\begin{abstract}
Time to culture conversion in smokers with pulmonary tuberculosis. J.A. Gullón, I. Suárez, M. Lecuona, R. Fernández, G. Rubinos, A. Medina, C. Cabrera, I. González. Background and objective. It has been reported that tobacco smoking slows the sterilisation of sputum culture in pulmonary tuberculosis, but the factors that could delay culture conversion in patients who smoke are not known. Our aim is to identify the factors influencing sputum culture conversion in smokers with pulmonary tuberculosis.

Methods. Ninety-nine patients with a smoking history and diagnosed with pulmonary tuberculosis were analysed retrospectively. The relationship between sputum culture status at the second month and the following variables: age, gender, pack-years index, comorbid diseases, number acid-fast bacilli (AFB) in sputum smear examination, radiological findings (cavitary, extensive or limited disease),
\end{abstract}

drug susceptibility pattern and initial treatment, was analysed. The Student $t$-test, $c h i$-square test and logistic regression model with forward stepwise conditional methods were used for statistical analysis. A $p$ value of $<\mathbf{0 . 0 5}$ was considered to be statistically significant.

Results. Twenty six patients $(26.2 \%)$ : 18 males $(22.2 \%)$ and 8 females $(44 \%)$ were sputum culture positive at the end of the second month of treatment. In univariate analysis, culture conversion time was significantly associated with female gender and extensive disease, but in a logistic regression analysis was only correlated with female gender $(\mathrm{OR}=5.6395 \%$ CI 1.21-20.64- $p=0.02)$.

Conclusion. In current smokers with pulmonary tuberculosis, the 'time to culture' conversion relates only to the female gender.

Monaldi Arch Chest Dis 2009; 71: 3, 127-131.

Keywords: Culture conversion, Gender, Pulmonary tuberculosis, Smoking.

1 Pneumology Service, Hospital Universitario de Canarias, La Laguna (Santa Cruz de Tenerife),

2 Microbiology Service, Hospital Universitario de Canarias, La Laguna (Santa Cruz de Tenerife), Spain.

Correspondence: José Antonio Gullón, C/ La Marina n49 4 izquierda, 38001; Santa Cruz de Tenerife, Spain; e-mail: jagb965@yahoo.es

\section{Introduction}

The most widely accepted measure of treatment response in patients with pulmonary tuberculosis (TB) is the disappearance of acid fast bacilli (AFB) from both sputum smear and culture. The number of bacilli decreases rapidly after the start of treatment and in three weeks approximately 80$85 \%$ of sputum smears become negative for AFB [1], but in some patients, even without drug resistance, the time to sputum sterilisation is longer. Furthermore, of the multiple risk factors of relapse that have been studied, the most important diseaserelated factor is probably the presence of positive culture on completion of the 2-mo initial phase of therapy $[2,3]$.

Tobacco smoking has increased substantially in developing countries over the past three decades and it is in these countries that most of the morbidity and mortality due to TB occurs. Nowadays, there is strong evidence that smokers, when compared with people who do not smoke, have an increased risk of contracting active TB and it being fatal, but there is insufficient evidence to support an association between smoking and other outcomes such culture conversion [4-6], although there are a few studies that discuss this topic purely in relation to smokers.
With this in mind, we performed an observational study to identify the factors influencing sputum culture conversion in smokers with pulmonary tuberculosis.

\section{Material and methods}

\section{Patients}

We retrospectively analysed all patients, current smokers, with pulmonary TB diagnosed in our Department between May 1997 and December 2007. All cases were negative in the HIV serologic test.

The exclusion criteria were as follows: resistance to more than one first-line antituberculosis (antiTB) drugs, patients unable to collect sputum smears after beginning treatment, patients who did not attend their scheduled appointment or died during antiTB treatment.

We used Ziehl-Neelsen staining to document AFB in sputum smear. The diagnosis was made by isolating $M$. tuberculosis in liquid culture medium BACTEC ${ }^{\circledR}$ MGITTM 960 (Becton Dickinson, Sparks, USA) and/or Lowenstein-Jensen solid medium (bioMérieux, France). Cultures were incubated at $37^{\circ} \mathrm{C}$ for 42 and 60 days, respectively. The species having positive cultures were identi- 
fied by DNA hibridation with genetic probes (Accuprobe $^{\circledR}$, Gen Probe, San Diego, LA, USA) and by conventional methods.

Patients started antiTB treatment according to the guidelines of the Spanish Society of Pulmonology and Thoracic Surgery (SEPAR) [7] in a 6-mo regimen with isoniazid $(\mathrm{H})$, rifampin $(\mathrm{R})$, pirazinamide $(\mathrm{Z})$, with or without ethambutol (E), when the first positive smear was detected.

The Canetti, Rist and Grosset method (BMérieux, France) was used to study sensitivity to the following antimicrobials: $\mathrm{H}(0.2$ and 1 $\mu \mathrm{g} / \mathrm{ml})$, streptomycin (4 and $10 \mu \mathrm{g} / \mathrm{ml}), \mathrm{E}$ ( 2 and 3 $\mu \mathrm{g} / \mathrm{ml}), \mathrm{R}$ (20 and $40 \mu \mathrm{g} / \mathrm{ml})$, PAS (0.5 and 1 $\mu \mathrm{g} / \mathrm{ml})$ and $\mathrm{Z}(200 \mu \mathrm{g} / \mathrm{ml})$.

Sputum smear test was performed at the first, second, fourth and sixth month after antiTB treatment commenced.

This evaluation was approved by our Investigational Review Board and it was deemed that patient consent was not necessary when the study started.

\section{Variables}

The following variables were recorded: age, gender, pack/year index, comorbid diseases, sputum smear examination, radiological findings, drug susceptibility pattern and initial treatment. All these variables were correlated with the results of sputum culture in liquid mediun at second month after starting antiTB treatment.

Radiologic findings were classified as limited or extensive disease.

Sputum smear was graded as follows: no $\mathrm{AFB} / 300$ fields (negative), 1-9 AFB/100 fields (+), 1-9 AFB/10 fields (++), 1-9 AFB/field (+++) and $>9 \mathrm{AFB} /$ field (++++) [8].

Initial treatment was codified as 3 or 4 , according to the number of drugs employed.

\section{Definitions}

The following definitions were established: $e x$ tensive disease as infiltrates involving at least $75 \%$ of lung fields or presence of cavities totalling at least $15 \mathrm{~cm}$ in diameter [9], initial treatment as the therapy initiated when the first sputum smear or culture was positive.

\section{Statistical analysis}

Quantitative variables were expressed as means and qualitative variables as percentages. Student's $t$-test was used to compare quantitative variables and chi-square distribution for qualitative variables.

A logistic regression model with forward stepwise conditional methods was used to identify factors that could have influenced culture conversion. Persistent sputum culture positivity at the end of 2 months of treatment was a dichotomus dependent variable.

A $p$ value of $<0.05$ was considered to be statistically significant.

\section{$\begin{array}{ll}\text { Results } & \text { Rerall Results }\end{array}$}

During the study period, 102 patients with smoking history were diagnosed with TB, 7 of whom met at least one of the exclusion criteria. The final study sample consisted of 95 patients with 33.05 pack/year index SD 19.98, mean age 44.2 years SD 14.75, 77 were males and 18 females. 26 patients $(27.3 \%)$ had concomitant diseases, diabetes mellitus, found in 16 patients, being the most frequent. Four patients had a history of injection drug use and 30 had a history of alcohol consumption, although alcohol abuse was recorded only in three patients.

AFB were identified in sputum smear in 76 cases $(80 \%)$ and treatment was started with three drugs in 85 patients $(89.4 \%$ ). 91 patients were treated by self-administered therapy and four by directly observed therapy (DOT) and treatment outcome according with WHO indicators was: cured in 94 and default in one. A drug susceptibility test was available in all cases and resistance was found in nine $(9.4 \%)$ sputum culture isolates, six being resistant to $H$, two to $R$ and one to $S$.

The disease was classified as extensive in 32 patients $(33.6 \%)$.

General characteristics of the patients according to gender are shown in table 1 .

\section{Culture conversion}

Sputum culture was positive after one month in 36 patients (37.8\%): 27 males (35\%), 9 females $(50 \%)$, and after two months in 26 (27.3\%): 18 males $(23.3 \%)$ and 8 females (44.\%). Figure 1 represents the 'distribution over time' of culture conversion.

As shown in table 2, in univariate analysis, time to culture conversion was significantly associated with female gender $(p=0.03)$ and extensive disease $(p=0.01)$.

In logistic regression analysis the likelihood of persistent culture positivity on completion of the 2-mo initial phase of therapy was only correlated with female gender (table 2).

\section{Discussion}

Drug treatment of active pulmonary tuberculosis is a key factor in interrupting the transmission of this disease. Although the specificity of AFB in sputum in the diagnosis of TB is over $98 \%$, it remains a poor measure of infectivity because in some patients with negative smears, bacilli can be detected in culture medium and these cases may be responsible for transmission of at least one in six patients with pulmonary involvement [10], so it would be very important to identify factors that could delay culture conversion. In this sense, factors such as the number of AFB in initial sputum smear, extensive cavitary disease [11-12] and the number of drugs initially employed [13] have been studied well.

There is limited data available to associate smoking and culture conversion but it is well recognised that tobacco consumption and TB are 
Table 1. - General characteristics according to gender

\begin{tabular}{|c|c|c|c|}
\hline & Male & Female & $\mathbf{P}$ \\
\hline $\begin{array}{c}\text { Age (years) } \\
<30 \\
30-50 \\
>50\end{array}$ & $\begin{array}{l}15(19.5 \%) \\
37(48.1 \%) \\
25(32.4 \%)\end{array}$ & $\begin{array}{c}4(22.2 \%) \\
10(56.6 \%) \\
4(22.2 \%)\end{array}$ & 0.7 \\
\hline Pack - years index & $34.81 \pm 20.83$ & $22.75 \pm 9.11$ & 0.002 \\
\hline Comorbid diseases & $24(31.1 \%)$ & $2(11.1 \%)$ & 0.08 \\
\hline $\begin{array}{l}\text { Radiological findings } \\
\text { extensive disease }\end{array}$ & $23(28.3 \%)$ & $9(50 \%)$ & 0.07 \\
\hline $\begin{array}{l}\text { Sputum smear (AFB) } \\
\quad \text { negative } \\
+ \\
++ \\
++ \\
++ \\
++++\end{array}$ & $\begin{array}{l}17(23.3 \%) \\
5(6.5 \%) \\
6(7.8 \%) \\
10(12.9 \%) \\
39(50.6 \%)\end{array}$ & $\begin{array}{l}2(11.1 \%) \\
2(11.1 \%) \\
2(11.1 \%) \\
3(16.7 \%) \\
9(50 \%)\end{array}$ & 0.5 \\
\hline Drug resistance & $7(9.1 \%)$ & $2(11.1 \%)$ & 0.5 \\
\hline $\begin{array}{c}\text { Initial treatment } \\
3 \text { drugs } \\
4 \text { drugs }\end{array}$ & $\begin{array}{l}70(90.9 \%) \\
7(9.1 \%)\end{array}$ & $\begin{array}{l}15(83.3 \%) \\
3(16.7 \%)\end{array}$ & 0.2 \\
\hline
\end{tabular}

AFB: acid-fast bacilli.

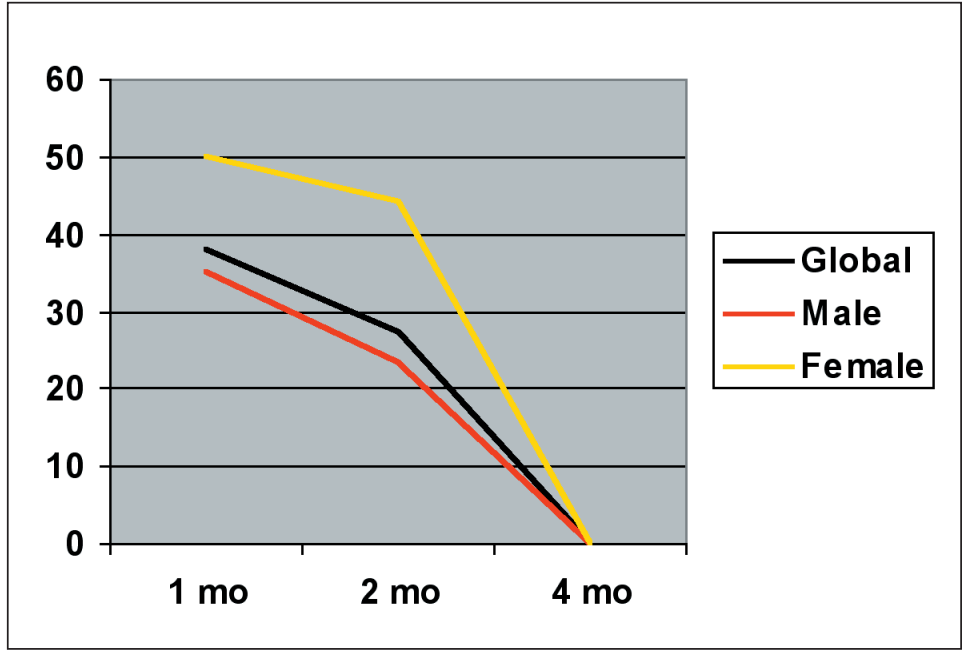

Fig. 1. - Distribution over time of culture positivity in percentage (\%). status, no culture, at second month of treatment was correlated with the number of AFB in smear sputum and foreign-born patients, although these results could not be verified in female smokers since only two females were included. The influence of gender on culture conversion in the present study may be due to higher frequency of extensive disease at presentation in women, as shown in table 1 , but when both variables were included in the logistic regression analysis a correlation was only established with gender, thus we believe that delayed conversion is specifically related to sex but not to the extension of the disease.

As in other studies [12-13] no association was found between drug resistance and culture conversion.

An important finding of the present

highly prevalent in many countries, especially in developing countries where the percentage of patients with smoking history is around 54\% [14]; therefore the interaction between tobacco and TB epidemics is an important public health issue. Therefore further investigation into the factors that prolong the infectivity period in smokers with TB disease could be of interest.

In this study the above factors were analysed in patients with current smoking history, only the female gender being found to be independently associated with culture conversion. Few studies have addressed this topic. However, Abal et al [15] in a sample of one hundred and eighty-three current smokers with pulmonary TB reported that the sputum smear study is that around one half of the women smokers with pulmonary TB had a longer time to culture conversion and this could have implications for transmission and relapse of the disease; therefore medical advice and counselling in smoking need to be reinforced in this population. Such activity would be more important in low-income countries as well as our own country which, together with Portugal, has the highest incidence rates of TB in Western Europe.

The mechanisms behind the link between smoking and TB are unclear. A large body of scientific evidence suggests that cigarette smoke inpha by macrophages in the lung, which plays a role hibits the production of tumour necrosis factor-al- 
Table 2. - Univariate and logistic regression analysis for factors associated with delayed culture conversion

\begin{tabular}{|c|c|c|c|c|c|}
\hline & \multicolumn{2}{|c|}{ Univariate and $p$ values } & \multicolumn{3}{|c|}{ Logistic regresion } \\
\hline & DC & $\mathbf{p}$ & OR & $95 \% \mathrm{CI}$ & $\mathbf{p}$ \\
\hline \multicolumn{6}{|l|}{ Age } \\
\hline$\leq 30$ & $5(26.3 \%)$ & 0.6 & Ref & & \\
\hline $31-50$ & $15(31.9 \%)$ & & 0.10 & $0.006-1.75$ & 0.1 \\
\hline$>50$ & $6(20.6 \%)$ & & 0.52 & $0.12-2.15$ & 0.3 \\
\hline \multicolumn{6}{|l|}{ Gender } \\
\hline Male & $18(23.3 \%)$ & 0.03 & Ref & & \\
\hline Female & $8(44 \%)$ & & 5.63 & $1.21-20.64$ & 0.02 \\
\hline \multicolumn{6}{|c|}{ Pack-year index } \\
\hline$\leq 20$ & $8(24.1 \%)$ & 0.3 & Ref & & \\
\hline$>20$ & $18(34.6 \%)$ & & 1.35 & $0.40-5.76$ & 0.6 \\
\hline \multicolumn{6}{|c|}{ Extensive disease } \\
\hline no & $12(19.4 \%)$ & 0.01 & Ref & & \\
\hline yes & $14(43.7 \%)$ & & 1.81 & $0.54-5.99$ & 0.3 \\
\hline \multicolumn{6}{|l|}{ AFB smear } \\
\hline 0 & $0(0 \%)$ & 0.6 & Ref & & \\
\hline +/++ & $5(35.7 \%)$ & & 0.57 & $0.64-1.35$ & 0.6 \\
\hline$+++/++++$ & $21(34.4 \%)$ & & 1.15 & $0.06-5.41$ & 0.1 \\
\hline \multicolumn{6}{|c|}{ Diabetes mellitus } \\
\hline no & $21(25.3 \%)$ & 0.08 & Ref & & \\
\hline yes & $5(31.2 \%)$ & & 1.67 & $0.46-5.96$ & 0.4 \\
\hline \multicolumn{6}{|l|}{ Treatment } \\
\hline 3 drugs & $23(27 \%)$ & 0.7 & 1.20 & $0.19-3.49$ & 0.7 \\
\hline 4 drugs & $3(30 \%)$ & & Ref & & \\
\hline \multicolumn{6}{|l|}{ Resistance } \\
\hline no & $22(25.6 \%)$ & 0.5 & Ref & & \\
\hline yes & $4(44.4 \%)$ & & 1.23 & $0.53-3.49$ & 0.3 \\
\hline
\end{tabular}

DC: delayed culture conversion.

in containing the intracellular growth of M. Tuberculosis, and also reduces macrophage apoptosis which promotes the killing of mycobacteria [16]. Other potential mechanisms by which smoking may attenuate mycobactericidal activity include oxidative stress mechanic disruption of cilia function and other clearance mechanisms in the tracheobronchial system. Finally, it has been shown that time culture conversion is related to vitamin D receptor gene polymorphisms [17, 18]. All the above would cause a slow response to antimicrobials, but unfortunately it has still not been demonstrated that these changes are more prevalent in women smokers and whether they reflect a greater sensitivity to the effects of tobacco smoke as has been shown in other diseases such as lung cancer and chronic obstructive pulmonary disease. This could prove interesting for future research.

The present study has certain limitations. First, the sample size is small, therefore our results must be interpreted with caution. Second, we can not ensure treatment adherence because in the majority of cases the treatment was self-administered and DOT was only offered to "likely defaulters", but we believe that its influence in final results is lim- ited because our group of patients for their characteristics (no HIV, no history of homelessness, low frequency of injection or noninjection drug use or alcohol abuse, no incarceration and all attending routine appointments) had a low risk of nonadherence [19] and, in randomised controlled trials, the completion and cure rates of treatment $[20,21]$ and the proportion of negative sputum cultures after two months [22] were not lower among self-administered patients than among those on directly observed therapy. Furthermore, several aspects that promote adherence should be emphasised: more than $90 \%$ of antimicrobials were given together in the same pill and it should be borne in mind that our National Health System ensures universal free coverage with totally or partially financed treatment; other authors have suggested that in our country is possible to obtain good completion rates without resorting to DOT in all patients [23].

In addition, it could be surprising that about $89.4 \%$ of patients were treated with three drugs, but as we mentioned above the initial treatment regimen was decided according to the guidelines of Spanish Society of Pulmonology and Thoracic 
Surgery and it has been suggested that it is very likely that an initial regimen without $E$ would be a valid treatment option for most Spanish born-patients because $\mathrm{E}$ is included in standard regimen to cover the possibility of high primary resistance to $\mathrm{H}$ and the prevalence of this in Spain has not yet been determined with precision [24]. Furthermore, the National Plan for Prevention and Control of Tuberculosis in Spain, recently published [25], establish that the regimen without $\mathrm{E}$ may still be valid in the regions of our country where the overall rate of resistance to $\mathrm{H}$ is under $4 \%$. In this sense, we want to emphasise that in a national multicentre study with 1493 cases the percentage of $\mathrm{H}$ resistance in native population was 3.5\% [26] and in our sample the proportion of immigrants was only a $5 \%$.

Finally, another potential limitation is the absence of a control group, but the real aim of our study is to analyse the factors influencing culture conversion in smokers and not the role of smoking in the speed of culture sterilisation; this issue has been discussed in a previous study of our group, recently published [27]. Nevertheless we have prospectively collected all cases of pulmonary TB diagnosed in our department and we have a historic control group of fifty-five patients non-smokers (27\% women). This group was homogeneous with respect to the the group of smokers in all variables studied and culture status at second month was not correlated with gender.

In conclusion, we found that in pulmonary TB in patients with smoking history, the time to culture conversion is related to female gender, although prospective studies on a larger scale are required to confirm this issue.

\section{References}

1. Jindani, Abert VR, Edwards EA, et al. The early bactericidal activity drugs in patients with pulmonary TB. Am Rev Respir Dis 1980; 121: 939-948.

2. Benator D, Bhattacharya M, Bozeman L, et al. Tuberculosis Trial Consortium. Rifapentine and isoniazid once a week versus rifampicin and isoniazid twice a week for treatment of drug-susceptible pulmonary tuberculosis in HIV-negative patients: a randomized clinical trial. Lancet 2002; 360: 528-534.

3. Chang $\mathrm{KC}$, Leung CC, Yew WW, et al. A nested casecontrol study on treatment-related risk factors for early relapse of tuberculosis. Am J Respir Crit Care Med 2004; 170: 1124-1130.

4. Slama K, Chiang CY, Enarson DA, et al. Tobacco and tuberculosis: a qualitative systematic review and metaanalysis. Int J Tuberc Lung Dis 2007; 11: 1049-1061.

5. Lin HH, Ezzati M, Murray M. Tobacco smoke, indoor air polution and tuberculosis: a systematic review and meta-analysis. Plos Medicine 2007; 4: e20.

6. Zellweger JP. Tobacco and tuberculosis. Monaldi Arch Chest Dis 2008; 69: 83-85.

7. Vidal R, Rey R, Espinar A, et al. Treatment and retreatment of tuberculosis. Working Group of TIR Area. SEPAR. Arch Bronconeumol 1996; 32: 463-74.

8. American Thoracic Society and the Center for Disease Control Prevention. Diagnostic standards and classification of tuberculosis in adults and children. Am J Respir Crit Care Med 2000; 161: 1376-95.

9. Globe M, Iseman MD, Madsen LA, et al. Treatment of 171 patients with pulmonary tuberculosis resistant to isoniazid and rifampin. $N$ Eng $J$ Med 1993; 328: 527-32.

10. Hernández-Garduno $\mathrm{E}$, Cook $\mathrm{V}$, Kunimoto $\mathrm{D}$, et al. Transmission of tuberculosis from smear negative patients: a molecular epidemiology study. Thorax 2004; 59: 286-290.

11. Telzak EE, Fazal BA, Pollard CL, et al. Factor influencing time to sputum conversion among patients with smear-positive pulmonary tuberculosis. Clin Infect Dis 1997; 25: 666-670.

12. Güler M, Ünsal E, Dursun B, et al. Factors influencing sputum and culture conversion time among patients with new case pulmonary tuberculosis. Int J Clin Pract 2007; 61: 231-235.

13. Liu Z, Shilkret KL, Ellis HM. Predictors of sputum culture conversion among patients with tuberculosis in the era of tuberculosis resurgence. Arch Intern Med 1999; 159: 1110-1116.

14. Leung C.C, Yew WW, Chan CK, et al. Smoking and tuberculosis in Hong Kong. Int J Tuberc Lung Dis 2003; 7: 980-986.

15. Albal AT, Jayakrishnan B, Parwer S, et al. Effect of cigarette smoking on sputum smear conversion in adults with active pulmonary tuberculosis. Respir Med 2005; 99: 415-420.

16. Pai M, Mohan A, Dheda K, et al. Lethal interaction: the colliding epidemics of tobacco and tuberculosis. Expert Rev Anti Infect Ther 2007; 5: 385-391.

17. Roth DE, Soto G, Arenas F, et al. Association between vitamin $\mathrm{D}$ receptor gene polymorphins and response to treatment of pulmonary tuberculosis. J Infect Dis 2004; 190: 920-927.

18. Babb C, van der Merwe L, Beyers N, et al. Vitamin D receptor gene polymorphins and sputum conversion time in pulmonary tuberculosis patients. Tuberculosis 2007; 87: 295-302.

19. Jasmer RM, Seaman CB, González LC, et al. Tuberculosis treatment outcomes. Directly observed therapy compared with self-administered therapy. Am J Respir Crit Care Med 2004; 170: 561-566.

20. Zvarenstein M, Schoeman JH, Vundule C, et al. Randomised controlled trial of self-supervised and directly observed treatment of tuberculosis. Lancet 1998; 352: 1340-1343.

21. Walley JD, Khan MA, Newell JN, et al. Effectiveness of the direct observation component of DOTS for tuberculosis: a randomised controlled trial in Pakistan. Lancet 2001: 354: 664-669.

22. Kamolratanakul P, Sawer H, Lertmaharit S, et al. Randomised controlled trial of directly observed therapy for patients with tuberculosis in Thailand. Trans $R$ Soc Trop Med Hyg 1999; 93: 552-557.

23. Caylá JA, Caminero JA, Rey R, et al. Working Group on Completion of Tuberculosis Treatment in Spain. Current status of treatment completion and fatality among tuberculosis patients in Spain. Int J Tuberc Lung Dis 2004; 8: 458-464

24. Ruiz-Manzano J, Blanquer R, Calpe JL, et al. Diagnosis and treatment of tuberculosis. Arch Bronconeumol 2008; 44: 551-556.

25. Tuberculosis Working Group Incorporating Scientific Societies, Autonomous Communities and the Ministry of Health and Consumer Affairs. National Plan for Prevention and Control of Tuberculosis in Spain. Arch Bronconeumol 2009; 45: 148-153.

26. Characteristics of tuberculosis in immigrants. Eccute Study results. Arch Bronconeumol 2009; 45 (Espec Cong): 211.

27. Gullón J.A, Suárez I, Lecuona M, et al. Tobacco smoking and sputum smear conversión in pulmonary tuberculosis. Med Clin (Barc) 2007; 128: 565-8. 